\title{
Wake Characteristics of a TriFrame of Axial-flow Hydrokinetic Turbines
}

\author{
Saurabh Chawdhary ${ }^{\mathrm{a}}$, Craig Hill ${ }^{\mathrm{b}}$, Xiaolei Yang ${ }^{\mathrm{c}}$, Michele Guala $^{\mathrm{a}}$, Dean \\ Corren $^{\mathrm{d}}$, Jonathan Colby ${ }^{\mathrm{d}}$, Fotis Sotiropoulos ${ }^{1,1, \mathrm{c}, *}$ \\ ${ }^{a}$ Saint Anthony Falls Laboratory, University of Minnesota, Minneapolis, Minnesota 55455, \\ $U S A$ \\ ${ }^{b}$ Department of Mechanical Engineering, University of Washington, Seattle, Washington \\ 98105, USA \\ ${ }^{c}$ Department of Civil Engineering, College of Engineering and Applied Science, Stony Brook \\ University, Stony Brook, New York 11794. USA \\ ${ }^{d}$ Verdant Power Inc., The Octagon, 888 Main Street, New York, NY 10044, USA
}

\begin{abstract}
An effective way to develop arrays of hydrokinetic turbines in river and tidal channels is to arrange them in TriFrame ${ }^{T M 1}$ configurations where three turbines are mounted together at the apexes of a triangular frame. This TriFrame can serve as the building block for rapidly deploying multi-turbine arrays. The wake structure of a TriFrame of three model turbines is investigated using both numerical simulations and experiments. In the numerical part, we employ large-eddy simulation (LES) with the curvilinear immersed boundary method (CURVIB) for fully resolving the turbine geometry details to simulate intra-turbine wake interactions in the TriFrame configuration. First, the computed results are compared with experiments in terms of mean flow and turbulence characteristics with overall good agreement. The flow-fields are then analyzed to elucidate the mechanisms of turbine interactions and wake evolution in the TriFrame configuration. We found that the wake of the upstream TriFrame turbine exhibits unique characteristics indicating presence of the Venturi effect as the wake encounters the two downstream turbines. We finally compare the wakes of the TriFrame turbines with that of an isolated single turbine wake to further illustrate how the TriFrame configuration affects the wake characteristics and power production in an array of TriFrames.
\end{abstract}

Keywords: hydrokinetic, marine, turbine, energy, TriFrame

\footnotetext{
* Corresponding author

Email address: fotis.sotiropoulos@stonybrook.edu (Fotis Sotiropoulos)
}

${ }^{1}$ TriFrame is a trademark of Verdant Power Inc.

Preprint submitted to Renewable Energy

February 13, 2017

(C) 2017. This manuscript version is made available under the Elsevier user license http://www.elsevier.com/open-access/userlicense/1.0/ 


\section{Introduction}

Marine and hydrokinetic (MHK) resources are gaining much interest as an emerging source of renewable energy in recent years. One way to harness MHK energy from rivers and tidal streams is using current driven hydrokinetic tur-

5 bines, which are modular and scalable in nature. A turbine array is usually employed to maximize the power extraction from any MHK site. To reduce the installation and maintenance efforts of the turbines underwater, an effective way to develop arrays of hydrokinetic turbines in river and tidal channels is to arrange them in conjunction. To optimize the performance of turbine arrays, a better understanding of turbine wake characteristics and turbine-turbine wake interaction is needed. In this work, we will employ both numerical and experimental methods to investigate the wakes characteristics of three turbines mounted in a triangular frame (TriFrame) configuration (as shown in Fig. 1).

Many published works studied the nature of the turbulent wake downstream

15 of a hydrokinetic turbine $[1,2,3,4,5,6,7,8,9,10]$, as well as arrays of wind turbines and effects of intra-turbine spacing within arrays [11, 12, 13, 14, 15] using theoretical, experimental and/or computational tools. However, there have been relatively fewer studies on arrays of hydrokinetic turbines. A brief review of experimental and numerical efforts of turbine array studies are presented first.

20 Myers et al. [16] performed a set of scaled experiments to mimic an array of marine turbines in a laboratory setting. A porous disc was used to model the turbines in the experiments. They investigated several intra-turbine spacings in the array and concluded that wake interaction can result in undesirable effects of reduced power and increased fatigue loading for downstream turbines in the

25 array. Using similar tools, Daly et al. [17] investigated effects of marine turbines in a split tidal channel. Stallard et al. [18] studied the wake structure and recovery of multiple axial-flow hydrokinetic turbines in several configurations. Their experiments indicated that the wake recovers $80 \%$ at 10 rotor diameters downstream of the turbine. The effect of lateral spacing on the shape of the wake was also discussed.

Early computational efforts modeled MHK turbines in an array as single energy extraction points in a 2D domain. James et al. [19] used a modification of the Environmental Fluid Dynamics Code (EFDC) developed at Sandia National Laboratories (SNL) to simulate the changes to marine environments caused by

35 an array of MHK turbines. Harrison et al. [20] and Malki et al. [21] employed the Reynolds-Averaged Navier-Stokes (RANS) equations and the Blade Element Method (BEM) to simulate an array of tidal turbines, respectively. Harrison et al. [20] observed faster wake recovery when compared to the actuator disc parametrization of turbine. No comparison with experimental data was made 40 in both papers [20, 21]. Colby and Adonizio [22] at Verdant Power studied turbine-turbine interaction and its effect on marine ecology using ANSYS CFX. However, the simulations didn't resolve the full turbine wake and hence underpredicted the strength of wake. Bai et al. [23] also studied multi-row arrays of turbine wakes using Fluent with an actuator disc model but no validation of the 45 model was provided. Olczak et al. [24] assessed the accuracy of blade element 
method and RANS in predicting the thrust coefficient and wake velocities using commercial CFD package StarCCM+. It was found that the predictions worsens with the increasing number of turbines in the array with worst prediction for turbines in downstream rows of the array. The National Renewable Energy

50 Laboratory (NREL) created a framework for simulating MHK turbine arrays in natural waterways [25]. The effect of incoming turbulence on the wake characteristics was studied in an artificial straight channel. Ten different configurations including counter-rotating and co-rotating turbines modeled as actuator discs were investigated. It was found that for non-staggered co-rotating case, 55 increasing streamwise spacing between rows improved array performance. Staggering turbines downstream in the row also resulted in improved performance. However, the published numerical data were not verified with any experimental measurements. Yang et al. [26] developed a computational framework to perform large eddy simulation (LES) of MHK turbine arrays in natural waterways. The turbine blades were parameterized using actuator lines. This framework was employed to analyze the wake of aligned arrays of MHK turbines with various intra-turbine spacing in a straight channel flow. More recently, Stansby and Stallard [27] exploited the self-similarity of the wake in order to obtain optimized inter-turbine spacing in an array. The depth-averaged wake model of 65 turbines in an array was obtained by superposition of velocity-deficit for a single turbine wake obtained from measurements (and applying self-similarity [10]).

In the above mentioned computational works turbine parametrization was used to save computational cost involved in resolving the detailed geometry of a turbine. However, it was shown by Kang et al. [8] that the classic actuator 70 disc and actuator line models without a model for the nacelle cannot accurately predict the velocity deficit in the near wake, wake meandering and turbulence intensity in the far wake. The geometry-resolving model using the curvilinear immersed boundary (CURVIB) method, on the other hand, captures the turbine wake dynamics for both near- and far-wake regions, and the computed results 75 agree well with the measurements

In this work, we employ the same numerical method, i.e. LES with the CURVIB method resolving every geometrical details of the turbine, as in Kang et al. [8], together with experiments to study a TriFrame of turbines, or simply TriFrame, which is defined as a layout where three turbines are mounted 80 together at the apexes of a triangular frame (Fig. 1 (a)). The resulting arrangement is equivalent to two rows in a staggered fashion. The objective of this work is to study the wake characteristics of the three turbines in a TriFrame configuration and evaluate the feasibility of using such system as a building unit for turbine arrays.

85 This paper is organized in the following fashion. Section 2 describes numerical methods used to perform the computational portion of this work. In Section 3 , the experimental and computational setup is described. This is followed in Section 4 by discussion of the results obtained. Finally, Section 5 concludes the findings of this work. 


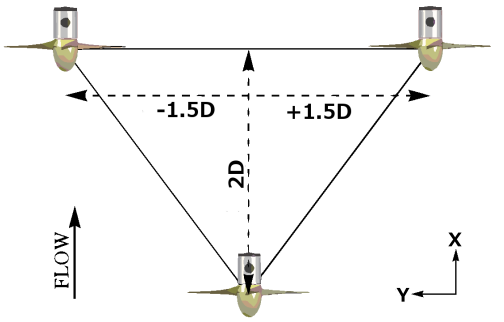

(a)

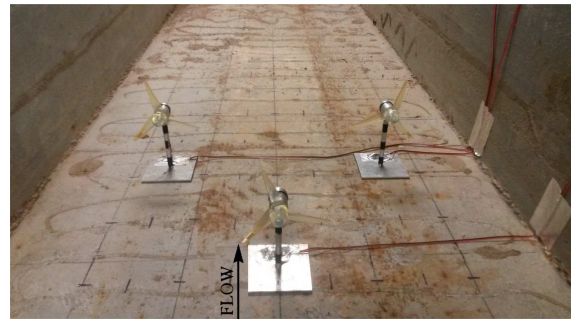

(b)

Figure 1: (a) Sketch of TriFrame geometry used in the experiments and computations $(\mathrm{D}=$ turbine diameter $=0.15 \mathrm{~m}$ ); (b) TriFrame of small scale model turbines placed in the laboratory flume at Saint Anthony Falls Laboratory (SAFL).

\section{Numerical Methods}

The CURVIB method $[28,29]$ is employed to simulate the turbulent flow past the TriFrame of axial flow turbines. The governing equations are the spatially filtered continuity equation (Eq. 2.1) and Navier-Stokes equation (Eq. 2.2) in generalized curviliear coordinates, which read as follows:

$$
\begin{gathered}
J \frac{\partial U^{i}}{\partial \xi^{i}}=0 \\
\frac{1}{J} \frac{\partial U^{i}}{\partial t}=\frac{\xi_{l}^{i}}{J}\left(-\frac{\partial}{\partial \xi^{j}}\left(U^{j} u_{l}\right)+\frac{1}{\rho} \frac{\partial}{\partial \xi^{j}}\left(\mu \frac{g^{j k}}{J} \frac{\partial u_{l}}{\partial \xi^{k}}\right)-\frac{1}{\rho} \frac{\partial}{\partial \xi^{j}}\left(\frac{\xi_{l}^{j} p}{J}\right)-\frac{1}{\rho} \frac{\partial \tau_{l j}}{\partial \xi^{j}}\right)
\end{gathered}
$$

95

where $\xi^{j}$ is the $\mathrm{j}^{\text {th }}$ curvilinear coordinate, $J$ is the Jacobian of the geometric transformation, $\xi_{l}^{i}$ are the transformation metrics, $g^{j k}$ is the contravariant metric tensor, $U^{i}$ are the contravariant volume fluxes, $u_{i}$ are the cartesian velocity components, $p$ is the pressure, $\mu$ is the dynamic viscosity, $\rho$ is the density and $\tau_{i j}$ is the subgrid-scale (SGS) stress tensor of the LES method. $U^{i}, u_{i}$ and $P$ are filtered quantities. Time averages of cartesian velocity components in the X, Y and $\mathrm{Z}$ directions are denoted as $\mathrm{U}, \mathrm{V}$ and $\mathrm{W}$ later in the discussion. Equations are expressed using Einstein's notation for tensors where repeated indices imply summation. The SGS stress $\left(\tau_{i j}\right)$ which appears after applying the spatial filter 105 to the curvilinear Navier-Stokes equations is modeled using the Smagorinsky model [30].

$$
\tau_{i j}-\frac{1}{3} \tau_{k k} \delta_{i j}=-2 \mu_{t} \overline{S_{i j}}
$$

where the eddy viscosity $\left(\mu_{t}\right)$ was further modeled by Smagorinsky as

$$
\mu_{t}=C_{s} \Delta^{2}|\bar{S}|
$$


In equations 2.3 and 2.4, the over-bar denotes a spatial filtering operation, $\overline{S_{i j}}$ is the filtered strain-rate tensor, $\delta_{i j}$ is Kronecker delta, $C_{s}$ is the Smagorinsky constant, $\Delta$ is the filter size (cube root of the grid cell volume in the present method)

and $|\bar{S}|=\sqrt{2 \overline{S_{i j} S_{i j}}}$. Smagorinsky constant $C_{s}$ is dynamically calculated using the method of Germano et al. [31]. More details of the $C_{s}$ calculation can be found in Kang et al. [29]. In the CURVIB method, the flow field is solved on a non-body-conforming grid (as shown in Fig. 3 (a)) while the immersed boundary is represented as an independent unstructured surface mesh (Fig. 3 (b)).

115 The background grid nodes are classified as either fluid nodes, solid nodes or Immersed Boundary (IB) nodes. The boundary conditions for the flow field simulations are specified by reconstructing the velocities on the IB nodes using the values on the neighboring fluid nodes and immersed boundary surface [32, 29]. Linear or quadratic interpolation can be employed for grids sufficiently fine to 120 resolve the viscous sublayer. If the first grid node off the boundary does not lie in the viscous region of the wall boundary layer near the immersed boundary, a power law wall model of Werner \& Wengle [33] (as implemented in Choi et al. [34],) is employed to reconstruct the velocities on the IB nodes. The equation for power law wall model is defined as:

$$
\frac{u}{u_{*}}= \begin{cases}z^{+} & : z^{+} \leq 12 \\ 8.3\left(z^{+}\right)^{\frac{1}{7}} & : z^{+}>12\end{cases}
$$

where $u_{*}$ is the shear velocity, $\nu$ is kinematic viscosity of water and $u$ is the wall-parallel velocity at $z$ distance from the wall and $z^{+}=z u^{*} / \nu$.

The governing equations are disretized in space using a second-order central finite difference scheme and advanced in time using a second-order fractional step method $[28,29]$. Iterative solvers implemented in PETSc (Portable, Ex130 tensible Toolkit for Scientific Computation) library are used for solving the discretized equations. Generalized Minimal Residual (GMRES) method [35] is used to solve the linear system for the pressure correction Poisson equation. Algebraic multigrid (AMG) is used as a preconditioner [29] for the GMRES method to accelerate the convergence. The non-linear discrete momentum equation is solved using matrix-free Newton-Krylov method. The inner iterations of the Newton-Krylov solvers also use GMRES method but without preconditioning. The code is efficiently parallelized using PETSc library and MPI (Message Passing Interface) to exploit massively parallel computer clusters. For more details on implementation of the numerical solvers, reader is referred to $[28,29]$.

Both near- and far-wake characteristics of a TriFrame of turbines are important for developing TriFrame based turbine arrays. However, it is very expensive to simulate both the near- and far-wake locations in a single simulation using a sharp interface immersed boundary method because of the additional computational cost from identifying fluid, IB and solid nodes at every time step in the IB method, and reconstructing the left-hand-side matrix in the Poisson solver. In order to reduce this computational cost, in this work we employ a domain splitting technique to simulate the near-wake and far-wake separately. 
In this technique, the velocities on a plane normal to the streamwise direction near the outlet of the near-wake simulation are saved at every time step. The saved velocity fields are then fed into the far-wake simulation as inflow conditions. The computational setup for the current simulations using this domain splitting technique will be presented in Section 3.2. Validation of this technique will be shown in Section 4.1.

\section{Test Case: TriFrame in Laboratory Flume} using a single turbine but with the same hydraulic condition was also completed. These data are compared to the wakes of a TriFrame turbine configuration. 


\subsection{Computational Setup}

isolated turbine for comparison. In order to save computational time, near-wake and far-wake simulations were carried out separately. The near-wake domain contains the turbines while the far-wake domain starts at the outflow of the near-wake domain as seen in Fig. 2. The streamwise length of the near-wake 195 and far-wake domains is $9 \mathrm{D}$ and $12 \mathrm{D}$, respectively. The velocity time-series from near-wake simulation is sampled at $x=8 D$ from the near-wake simulation and fed to the far-wake simulation. This amounts to a one-way coupling between the two computational domains. This one-way coupling is acceptable because convection of wakes to further downstream locations dominates the flows around by comparing flow-field in the overlapping region. The TriFrame of turbines is placed in the center of the flume such that the rotating center of the upstream T1 turbine (or the only turbine for single turbine case) is located at $(2 \mathrm{D}, 0$, 0.9D), 2D downstream from the inlet plane. The second row turbines (T2, T3) turbulent flow condition is prescribed at the inlet boundary. To achieve this, a separate precursor channel flow simulation is run with periodic boundary conditions in the streamwise direction to obtain a fully developed turbulent inflow. The cross section of this channel is the same as the flume. Time-series the inlet velocity boundary condition for the near-wake simulations. The inflow for the far-wake simulation, on the other hand, is provided by the outflow of the near-wake simulation using time series of velocity vector extracted at each point on the plane $X=8 D$ (marked as section AA' in Fig. 2) saved in the nearheight corresponding to the transition roughness regime. Since no roughness model is available to model this regime, the walls were assumed to be smooth. This assumption is not expected to significantly affect the velocity field near the turbines far away from the wall region. The top free surface of the channel, 220 in both near- and far-wakes simulations, was modeled as rigid lid. Since the free surface level in the experiments did not change more than $3.5 \%$ of the flow depth, the rigid lid assumption will be an acceptable modeling approach. At the exit of both near- and far-wake domains, Neumann boundary conditions are imposed. On the bottom bed and side walls, the first off-wall grid node was approximately 32 and 58 wall units away from the wall, respectively, in all cases. Since these points lie outside of the laminar region of the boundary layer, a wall-modeling approach was used (as described earlier in Section 2).

The size of computational domain for each simulation is presented in Table 1. The table also lists number of grid points $N_{x}, N_{y}$ and $N_{z}$ in the $\mathrm{X}, \mathrm{Y}$ 230 and $\mathrm{Z}$ directions, respectively. The grid was stretched such that points were clustered in the region of the turbines as well as immediately downstream of the turbines. Fig. 3 shows background grid for near-field simulation with TriFrame. Same figure also shows turbine represented by unstructured triangular mesh. In the near- and far-wake domains, the grid is stretched in the spanwise $(\mathrm{Y})$ and 


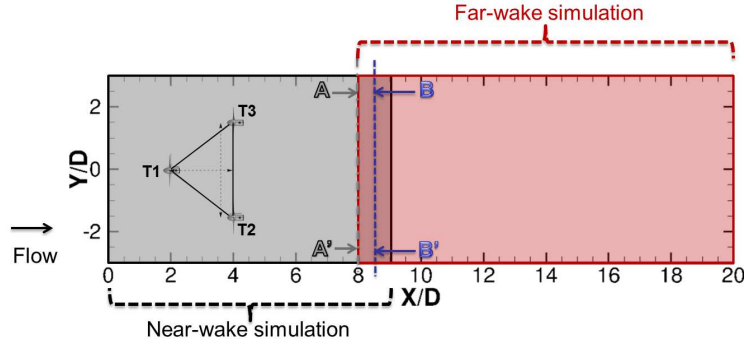

Figure 2: Simulation was performed in two parts - near-wake and far-wake. Section AA' is position where velocity was extracted in near-wake and fed to far-wake simulation. Comparison of velocity is made at section BB' for validation (see Fig. 4).

\begin{tabular}{ccccccc}
\hline Simulation & $\mathrm{X} / \mathrm{D}$ range & $\mathrm{Y} / \mathrm{D}$ range & $\mathrm{Z} / \mathrm{D}$ range & $N_{x}$ & $N_{y}$ & $N_{z}$ \\
\hline TriFrame: Near & {$[0,9]$} & {$[-3,3]$} & {$[0,1.87]$} & 592 & 521 & 184 \\
TriFrame: Far & {$[8,20]$} & {$[-3,3]$} & {$[0,1.87]$} & 601 & 521 & 184 \\
1 Turbine: Near & {$[0,9]$} & {$[-3,3]$} & {$[0,1.87]$} & 592 & 521 & 184 \\
1 Turbine: Far & {$[8,20]$} & {$[-3,3]$} & {$[0,1.87]$} & 601 & 521 & 184 \\
\hline
\end{tabular}

Table 1: Details of simulation grids used for TriFrame case and isolated single turbine (1 turbine) case. "Near" and "Far" denote near-wake and far-wake simulations, respectively.
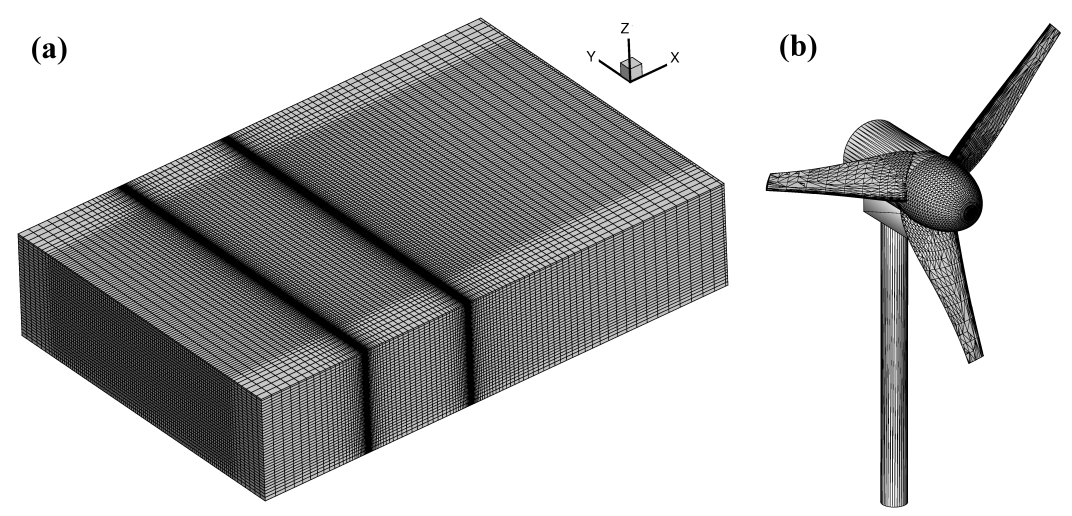

Figure 3: (a) Background grid for newar-wake simulation with TriFrame. Every fifth grid line is shown in all three direction. (b) Turbine geometry represented by unstructured triangular meshes. 

turbines. The $\mathrm{Y}$ spacing ranges between $\mathrm{D} / 100$ and $\mathrm{D} / 40$ whereas $\mathrm{Z}$ spacing range between $\mathrm{D} / 100$ and $\mathrm{D} / 75$ where minimum spacings occur close to turbine and maximum spacings occur away from the turbine. These values do not change in the streamwise direction for both near-wake and far-wake simulations. For

240 streamwise $(\mathrm{X})$ grid spacing in the near-wake simulation, the grid is stretched such that near the turbine, the X-spacing is D/400 so that the blade thickness is resolved by the background grid cells. Very far away downstream from the turbine location, this spacing grows to $\mathrm{D} / 25$. In the far-wake simulation, the $\mathrm{X}$-spacing has uniform value of D/50. For grid sensitivity studies, we rely on the immersed boundary method, the spatial resolution employed in the present work can give a reasonable agreement with the measurements of torque generation [4] and wake of the turbine [8]. The size of the time step was $\Delta t=7.3 \times$ $10^{-4} D / U_{b}$ for all simulations. Simulations were run until the total kinetic energy took approximately 1.5 - 2 flow-through times or $15-20$ rotor revolutions of the first (T1) turbine in TriFrame (or single turbine). Subsequently, the results were time averaged for another 180 rotor revolutions for the TriFrame nearwake simulation and 75 revolutions for the single turbine near-wake simulation.

255 For the far-wake simulations, averaging period was 180 rotor revolutions of T1 turbine in the TriFrame case and 63 rotor revolutions for single turbine case.

\section{Results and Discussion}

In the following section we discuss the results of the experiments performed at SAFL with a TriFrame of turbines and the subsequent LES. Both mean flow and turbulence statistics are presented below.

\subsection{Validation of domain splitting method}

The simulations were performed separately for near- and far-wake regions. The section between $8 D$ and $9 D$ is common between both simulations allowing for validation of the two-domain approach. Time averaged flow-field from both 265 near- and far-wake simulations is extracted at section BB' at $8.5 D$ (shown with blue dashed line in Fig. 2) and compared in Fig. 4. The contours of mean streamwise velocity from the two simulations match with each other well such that they differ by only $2 \%$ on average and $10 \%$ at most. Plots of mean velocity components and turbulence kinetic energy (TKE) in spanwise direction at the 270 hub height approach each other, confirming the validity of the two domain technique.

\subsection{Time-averaged flow field}

In Fig. 5, time-averaged streamwise velocity profiles are plotted from both the experimental measurements and LES prediction along the span of the do275 main in the hub height plane at different downstream locations. Velocity deficit 

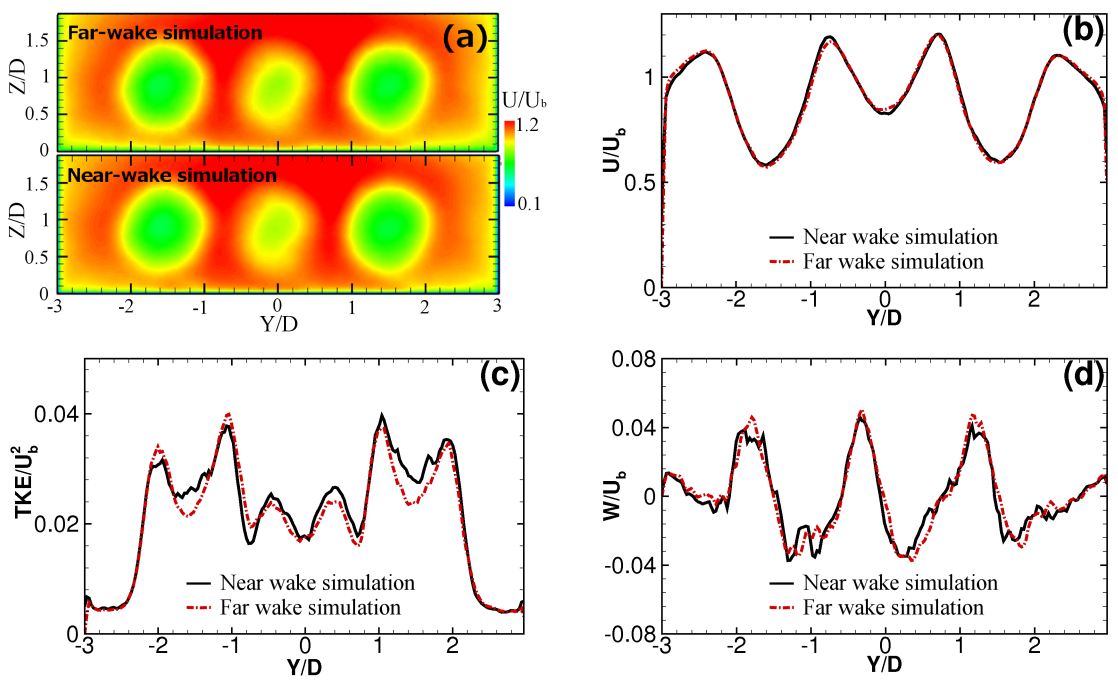

Figure 4: Comparison of flow field in section BB' of Fig. 2. (a) Normalized mean streamwise velocity $U / U_{b}$; (b) Spanwise profile of streamwise velocity at hub height; (c) Spanwise profile of TKE at hub height; (d) Spanwise profile of mean vertical velocity at hub height.

is created downstream of all three turbines. Simulation predictions show good agreement in the region downstream of the turbines. The peak in velocity deficit is captured accurately for both rows of turbines. Further downstream, the velocity is under-predicted by the LES. Velocity at the center line of the turbine wakes is within $7 \%$ of experimental value. Closer to the wall, towards the outer boundary of the wakes, the discrepancy is close to $10 \%$ which can be attributed to the discrepancy in the inlet profiles near walls.

The turbulence kinetic energy (TKE) in Fig. 6 shows a similar trend. Most of the TKE in the flow is generated due to the presence of the turbines. Similar to what is obtained in the measurement as well as observed by Kang et al. [8], the LES prediction shows multiple peaks in TKE created by each turbine. The peaks in TKE are well predicted in the near wake but under-predicted (by up to $25 \%$ ) in the far wake downstream region.

The vertical velocity component (due to the wake rotation) profiles in the same plane are plotted in Fig. 7. The incoming flow has little to no vertical velocity component at hub height. The flow past the first turbine near the blades has a significant vertical velocity component which is accurately predicted by LES. Further downstream, the vertical component weakens and the LES prediction is not as accurate as in the near wake. Unlike LES, the incoming 295 flow in the experiment has slight non-zero vertical velocity at hub height. This difference is propagated and seen downstream (until $\approx 1 D$ ). This difference aside, vertical velocity from LES compares well with the experiments.

Next, contours predicted by the simulations are plotted in a streamwisevertical plane normal to the channel bed and passing through the center of 


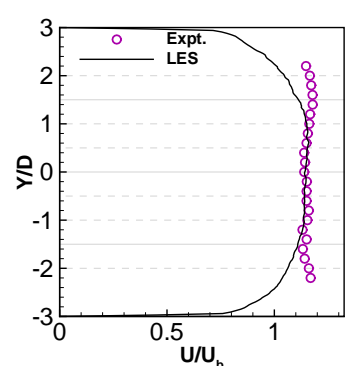

(a)

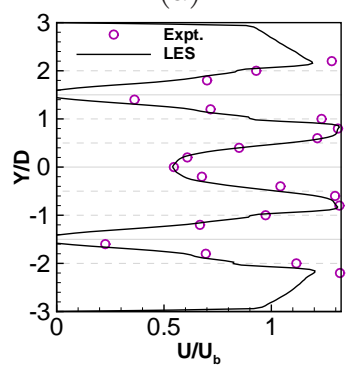

(d)

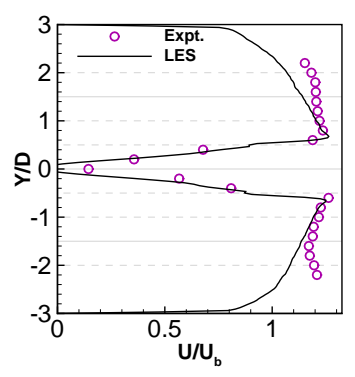

(b)

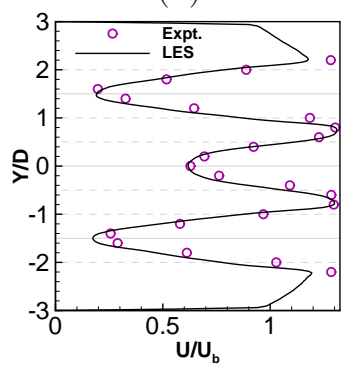

(e)

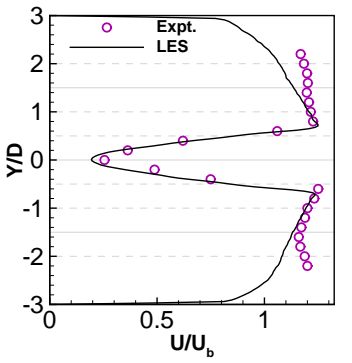

(c)

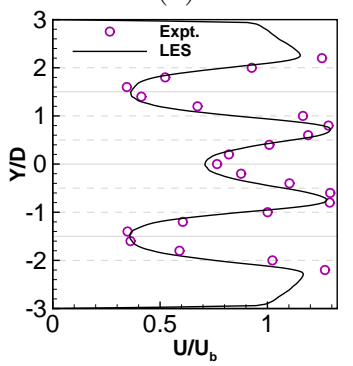

(f)

Figure 5: Comparison of streamwise velocity past the TriFrame of turbines at distances (a) $1 D$ upstream and (b) $0.5 \mathrm{D}$, (c) $1 \mathrm{D}$, (d) $2.5 \mathrm{D}$, (e) $3 \mathrm{D}$, (f) $4 \mathrm{D}$ downstream of the first turbine (T1) in a horizontal plane at the turbine hub height.

the turbines. In Fig. 8 the contours of time averaged streamwise velocity are shown for the three turbines of the TriFrame. Turbine numbers correspond to those indicated in Fig. 2. The contour plots show the shape of the wake and its recovery. There is a strong deceleration of the flow downstream of the turbine rotor and the hub. The wake is different for the first row and second row turbines with the latter showing lower recovery rate. The white lines on the plot mark zero streamwise velocity contour indicating presence of reverse flow in the vicinity of the hub. The transverse velocity contours in Fig. 9 show the compound wakes with an inner wake associated with the hub and an outer wake associated with the rotor. At approximately $2 D$ to $3 D$ downstream of turbine the two wake structures merge into one. The TKE (Fig. 10) shows contours similar to what was seen in Kang et al. [8]. Two regions of TKE generation exist - the tip of the rotor blades and the hub. These regions of TKE extend downstream, interacting close to the outer rotor shear layer. It is interesting to note that the wake of the upstream turbine shows significantly lower levels of TKE than the downstream ones.

To further analyze the differences between the wakes of different turbines in the TriFrame, contours of streamwise velocity and TKE are plotted on the wall parallel plane at hub height in Fig. 11. It is evident from this figure that the spanwise extent of the wake of the first turbine (T1) narrows starting at $2 D$ downstream from the turbine and recovers at a much higher rate than the wakes 


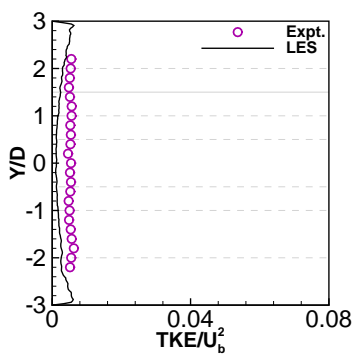

(a)

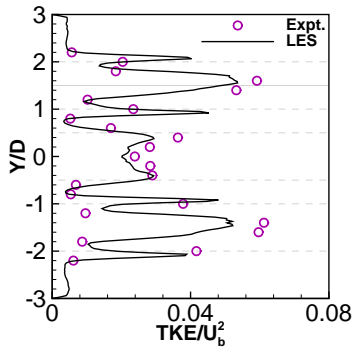

(d)

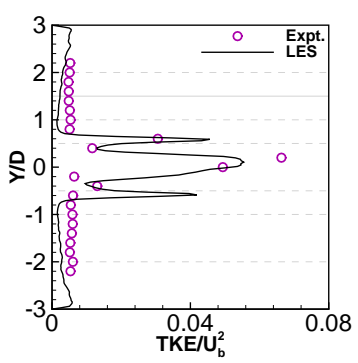

(b)

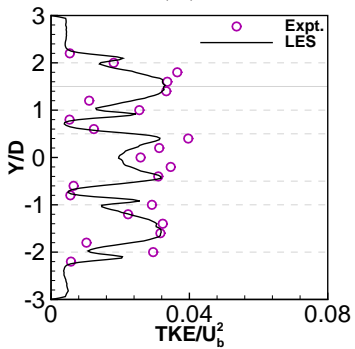

(e)

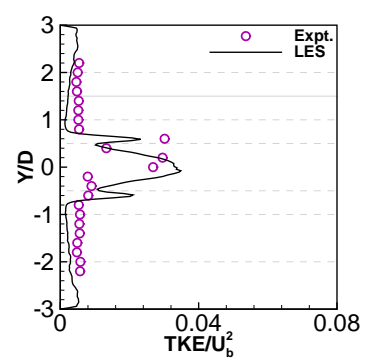

(c)

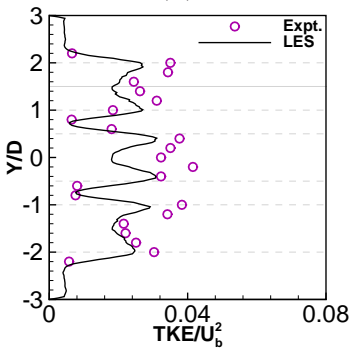

(f)

Figure 6: Comparison of turbulence kinetic energy (TKE) past the TriFrame of turbines at distances (a) $1 D$ upstream and (b) $0.5 D$, (c) $1 D$, (d) $2.5 D$, (e) $3 D$, (f) $4 D$ downstream of the first turbine (T1) in a horizontal plane at the turbine hub height.

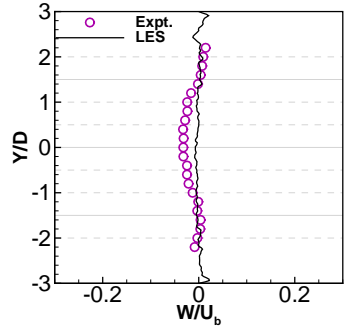

(a)

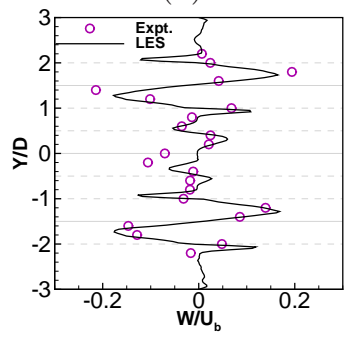

(d)

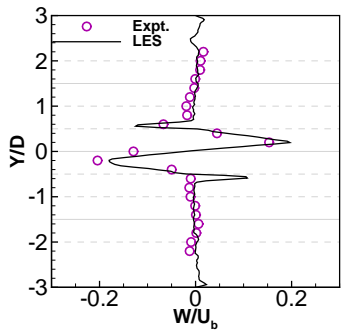

(b)



(e)

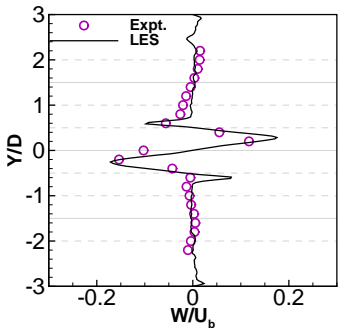

(c)

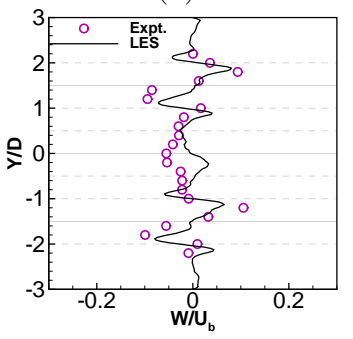

(f)

Figure 7: Comparison of vertical velocity past the TriFrame of turbines at distances (a) $1 D$ upstream and (b) $0.5 \mathrm{D}$, (c) $1 \mathrm{D}$, (d) $2.5 \mathrm{D}$, (e) $3 \mathrm{D}$, (f) $4 \mathrm{D}$ downstream of the first turbine (T1) in a horizontal plane at the turbine hub height. 


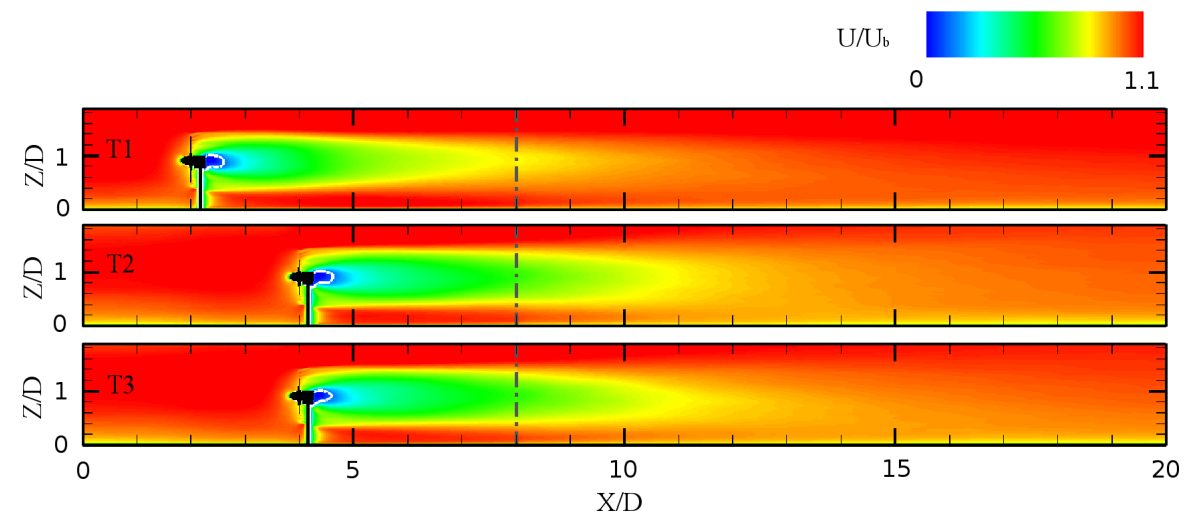

Figure 8: Contours of time averaged streamwise velocity, $U$, normalized by bulk mean inflow velocity, $U_{b}$, in the vertical plane passing through the center of the rotor for the turbines T1, $\mathrm{T} 2$ and T3. White line marks the contour of $U / U_{b}=0$. Dash-dot line shows the start of far-wake simulation.

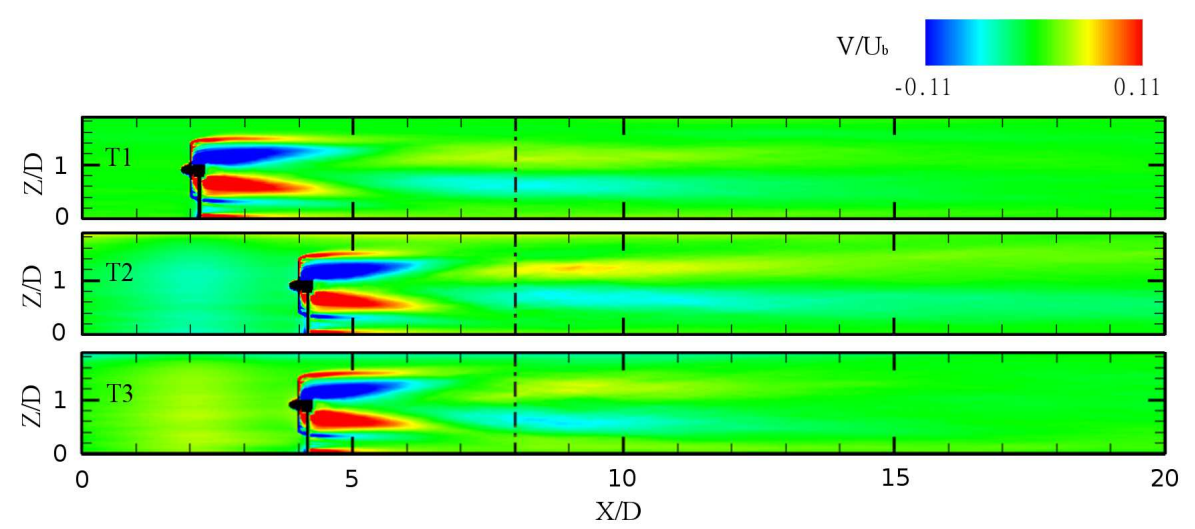

Figure 9: Contours of time averaged transverse velocity, $V$, normalized by bulk mean inflow velocity, $U_{b}$, in the vertical plane passing through the center of the rotor for the turbines $\mathrm{T} 1$, T2 and T3. Dash-dot line shows the start of far-wake simulation. 


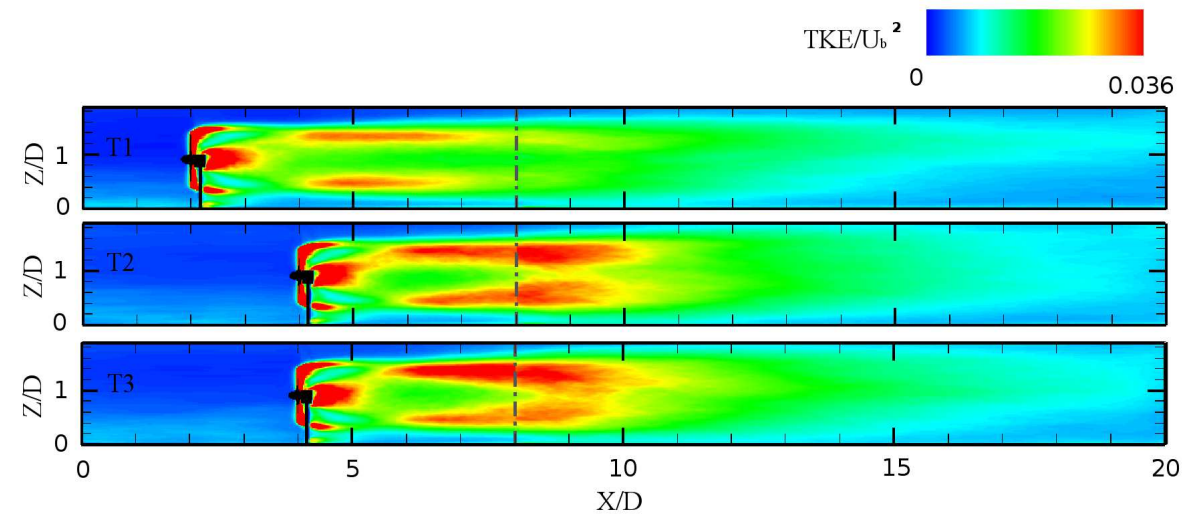

Figure 10: Contours of TKE normalized by the square of bulk mean inflow velocity in the vertical plane passing through the center of the rotor for the turbines T1, T2 and T3. Dash-dot line shows the start of far-wake simulation.

(a)

$\mathrm{U} / \mathrm{U}_{\mathrm{b}}$
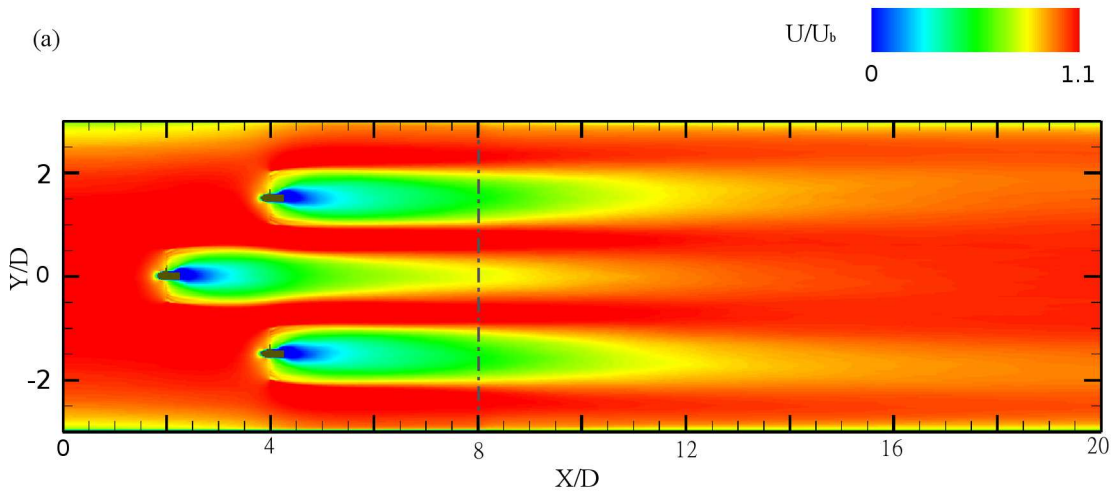

(b)
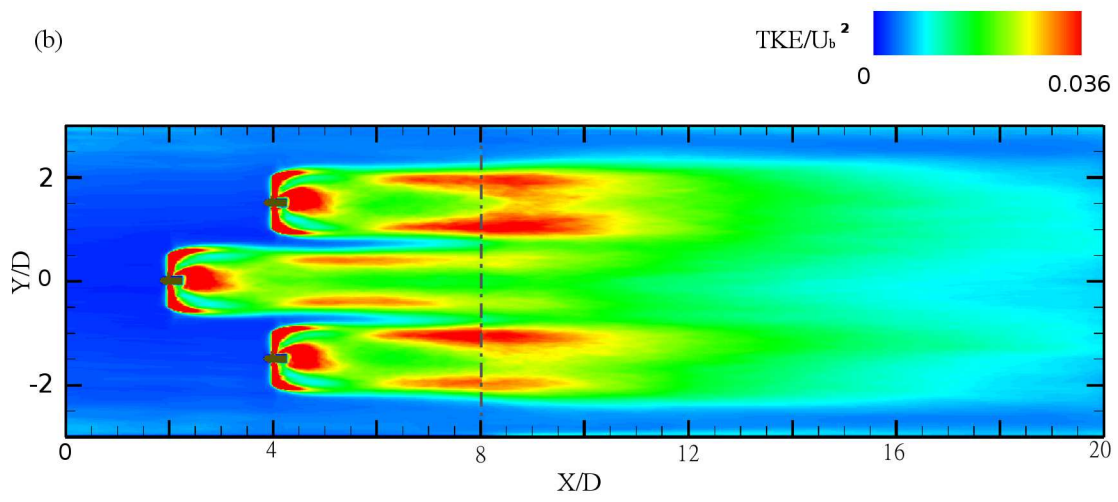

Figure 11: Contours of time averaged normalized (a) streamwise velocity and (b) TKE in hub height plane for the three turbines. Dash-dot line shows the start of far-wake simulation. 


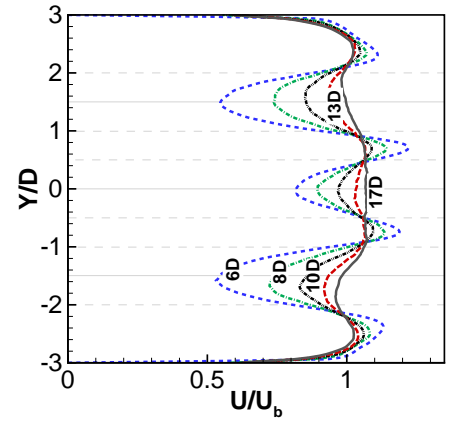

(a)

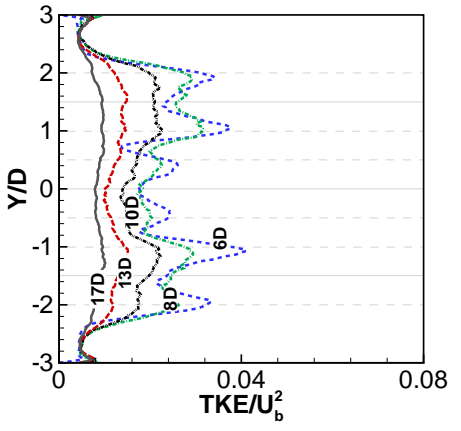

(b)

Figure 12: Profiles of (a) time averaged streamwise velocity and (b) TKE in far wake of TriFrame of turbine at several downstream distances (as labeled on plot) measured from the location of first row turbine (T1).

of the other two turbines. In this region of wake constriction, the streamwise velocity is higher and the TKE levels are lower than the two downstream turbine wakes. These findings, attributed to the Venturi effect induced by turbines T2 and T3, have also been reported in experiments of Chamorro et al. [13] on a laboratory scale staggered wind farm. Simulations of Ammara et al. [11] also observed flow acceleration between two turbines in staggered configuration in their simulations.

The spanwise variation of streamwise velocity and TKE for the far-wake simulations are shown in Fig. 12. In the previously discussed Fig. 5, large momentum deficits were observed in the near wake, specially within $0.5 \mathrm{D}$ of the turbines where the streamwise velocity is negative. In the far wake, after $10 \mathrm{D}$ downstream, most of the momentum has recovered and the velocity profile of the superwake of the TriFrame of turbines changes very little. Fig 12(b) shows that the TKE generated by turbines in the near wake (see Fig. 6) decays in the wake slowly. Beyond $10 D$, the TKE profiles of the superwake change very slowly.

\subsection{Comparison with single turbine wake}

To compare the wake of a TriFrame of turbines with that of a single turbine, a separate experiment and a separate LES were performed with an isolated single turbine in the same flume under the same conditions. The velocities from this experiment were also measured at hub height at different downstream locations in the near wake.

Fig. 13 and 14 illustrate the streamwise velocity and TKE, respectively, at certain distances downstream from the position of each of the three turbines in 345 TriFrame and the isolated single turbine. For T2 and T3 TriFrame turbines in plots of Fig. 13 and 14, positive Y represents the locations near the channel center and negative $\mathrm{Y}$ represents locations near the channel wall. This is not applicable to the other two turbines since they have symmetric wall conditions 


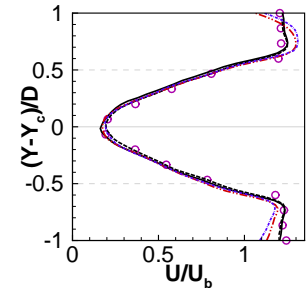

(a) $1 D$

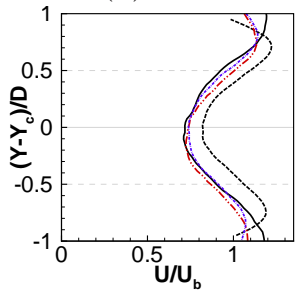

(e) $6 D$

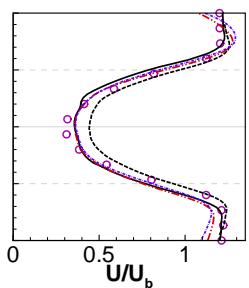

(b) $2 D$

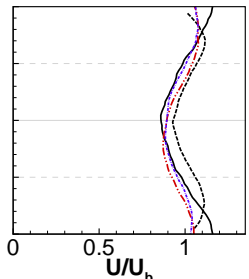

(f) $9 \mathrm{D}$

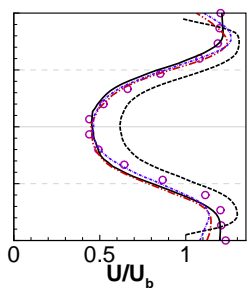

(c) $3 D$

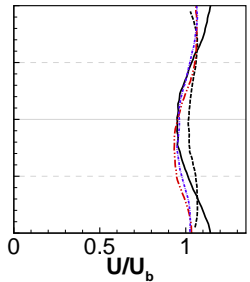

(g) $12 D$

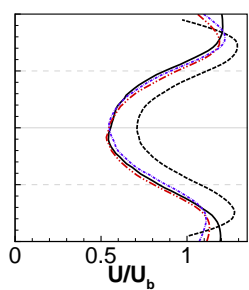

(d) $4 D$

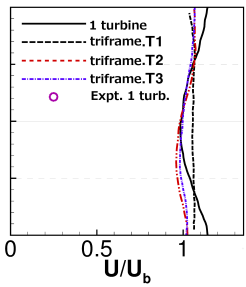

(h) $15 \mathrm{D}$

Figure 13: Comparing wakes past turbines in TriFrame with the wake past isolated single turbine. Streamwise velocity comparison downstream from the position of turbine in a horizontal plane at the turbine hub height (a) through $(\mathrm{h})$. 1 turbine is from isolated single turbine simulation and Expt. 1 turb. is from corresponding experiment. ( $Y_{c}$ denotes $Y$ coordinate of the center of the turbine.)

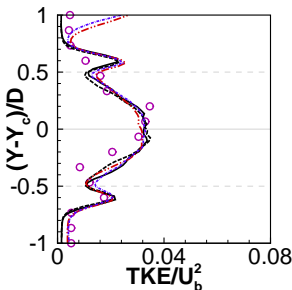

(a) $1 \mathrm{D}$

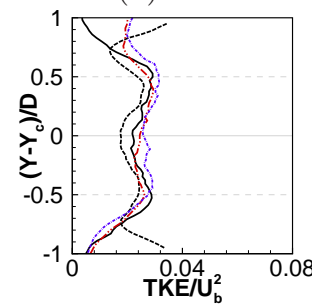

(e) $6 D$

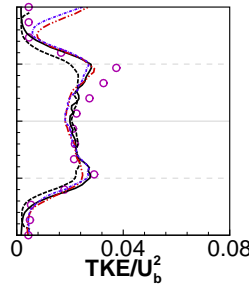

(b) $2 D$

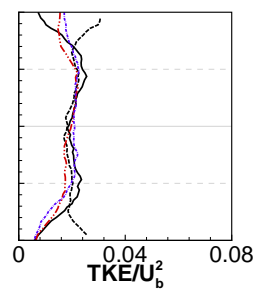

(f) $9 \mathrm{D}$

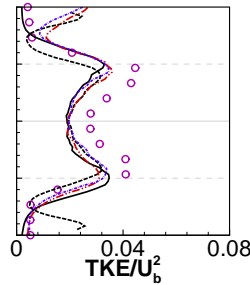

(c) $3 \mathrm{D}$

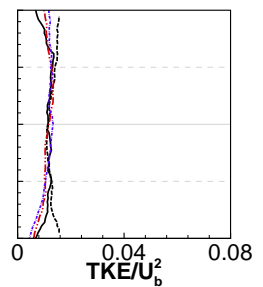

(g) $12 \mathrm{D}$

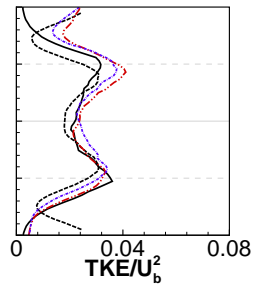

(d) $4 D$

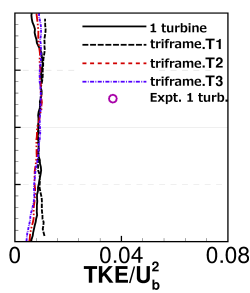

(h) $15 \mathrm{D}$

Figure 14: Comparing wakes past turbines in TriFrame with the wake past isolated single turbine. TKE comparison downstream from the position of turbine in a horizontal plane at the turbine hub height (a) through $(\mathrm{h})$. ( $Y_{c}$ denotes $\mathrm{Y}$ coordinate of the center of the turbine.) 


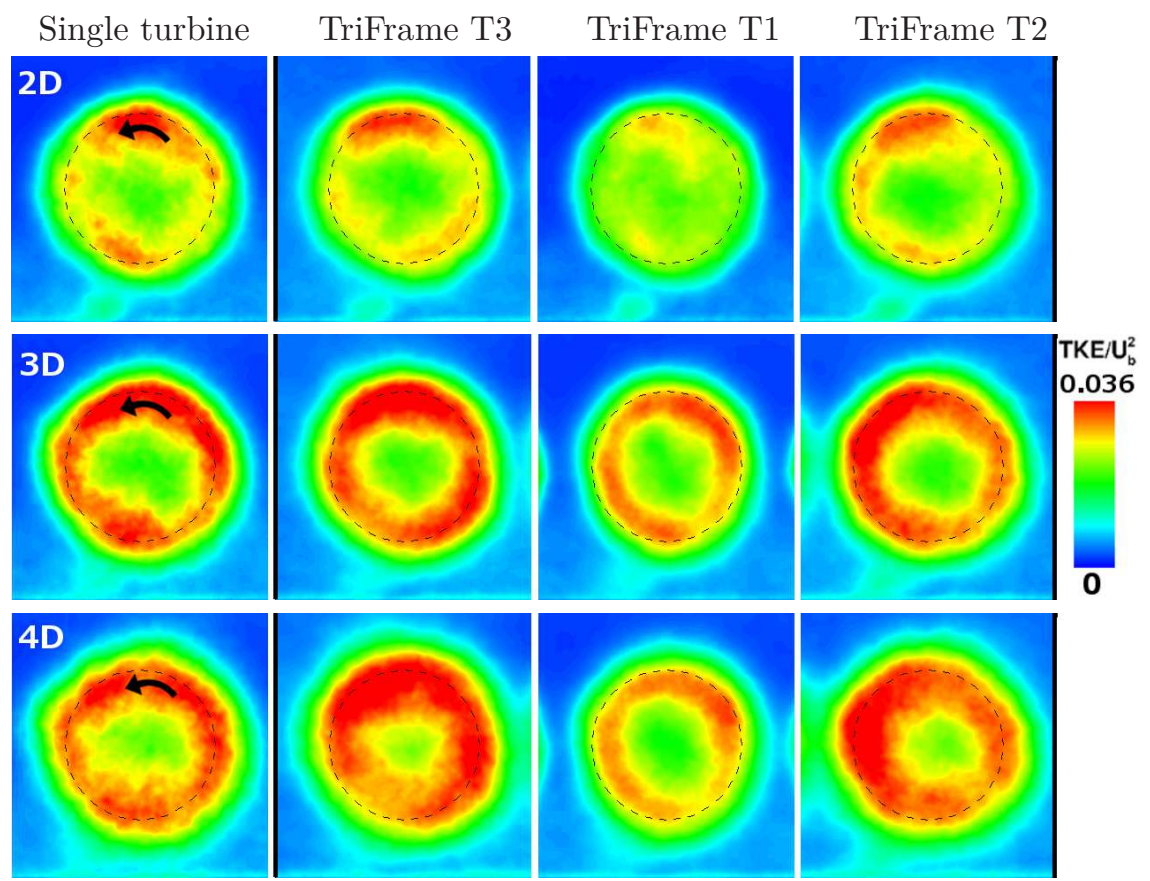

Figure 15: TKE contours on wall-normal (Y-Z) planes perpendicular to flow at $2 D, 3 D$ and $4 D$ downstream of turbines. Arrow shows direction of rotation for all turbines and dashed circle marks projection of area swept by turbine rotor. TriFrame turbines T1, T2 and T3 are as defined in Fig. 2. Channel side-walls are indicated by black lines on contour figures for T2 and T3. 
on both sides in the spanwise direction. As can be seen from Fig. 13, the streamwise velocity profile for all turbines look very similar at $1 D$ where the wake from the upstream turbine in the TriFrame is not yet influenced by the two downstream turbines. Further downstream ( $2 D$ to $3 D$ ), the wake of the upstream turbine T1 of the TriFrame recovers much faster than the isolated turbine and the two downstream TriFrame turbines because of the Venturi effect.

355 Beyond $3 D$ downstream, the wake recovery of the first turbine (T1) occurs at nearly the same rate as the other turbines. On the other hand, the spanwise profiles of velocity of the two downstream turbines of the TriFrame and the isolated single turbine still look very similar to each other. The difference between the T1 turbine wake and the other turbines diminishes as we move downstream.

360 At $12 D$ and beyond, this difference is very small and the mean wake for all turbines has mostly recovered. At $15 \mathrm{D}$, the $\mathrm{T} 1$ turbine wake has completely recovered. For the two downstream TriFrame turbines, the streamwise velocity is nearly symmetric within the wake, yet the streamwise velocity out of the wake is larger near the channel center than near the channel walls. The T2 and T3 365 turbines recover at a rate similar to that of the isolated turbine for the region within the wake i.e. within spanwise distance of approximately $0.5 \mathrm{D}$ centered at turbine rotor. In regions far from the center the recovery is faster for $\mathrm{T} 2$ and T3 turbines.

Lower TKE levels in the wake will result in less fatigue loading on the down370 stream turbines in the array. Therefore, it is important to discuss the spatial evolution of TKE in the wake. Fig. 14 highlights the spanwise profiles of TKE at different downstream locations, illustrating the effect of turbine-turbine interactions on TKE levels. After 2D, the TKE of the T1 turbine wake is significantly lower than both T2 and T3 turbines (between 17-23\% lower) and the isolated 375 turbine (23\% lower) in regions near the rotor tip (marked with dashed grey lines in the plots). In the inner wake region, the TKE levels for each of the turbines are comparable to each other for much of the wake except between $4 D$ and $8 D$ when T1 turbine inner wake also shows lower TKE (by 16-40\%) than the other turbines. The difference in TKE levels continues to diminish and profiles

380 of all wakes look similar at $12 \mathrm{D}$ and beyond where values are within $4 \%$ of each other. In Fig. 15 we plot the time-averaged TKE contours on Y-Z planes (wall-normal, perpendicular to flow) located at 2D, 3D and 4D downstream of the respective turbines First, the TKE from the first turbine in the TriFrame is lower than the other three turbines at all three downstream locations. At

$3852 \mathrm{D}$ and $3 \mathrm{D}$, the intensity of the maximum TKE from the two downstream turbines in the TriFrame are very similar to that of the single turbine. At 4D, on the other hand, the TKE in the wake of the two downstream turbines in the TriFrame are higher and distributed in a wider region, which is significantly different from that of the single turbine.

To understand the Venturi effects in the superwake, we define a special wake function $\Phi$ using the following relationship:

$$
\Phi(x, y)=\frac{U\left(x, y, z_{c}\right)-U\left(x, y_{c}, z_{c}\right)}{U_{h u b, \text { in }}}
$$




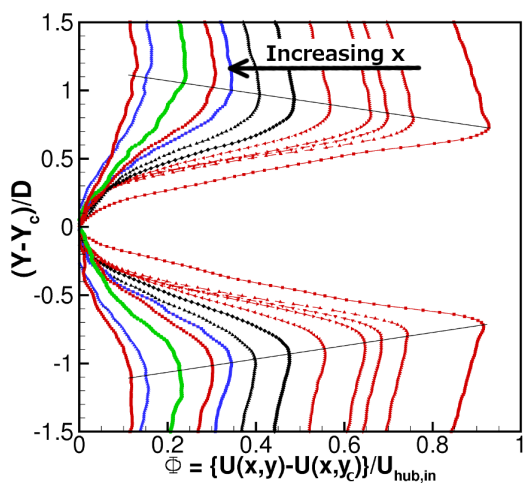

(a)

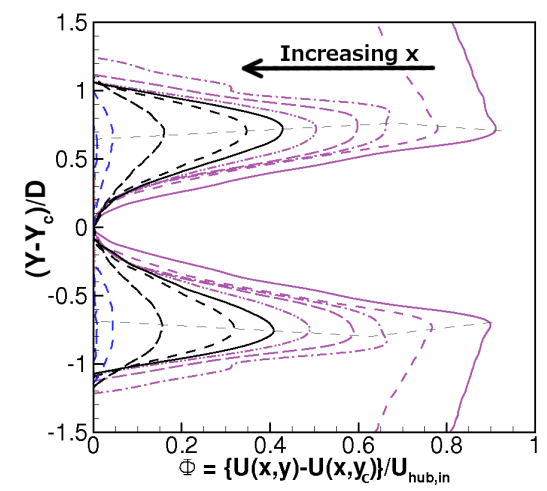

(b)

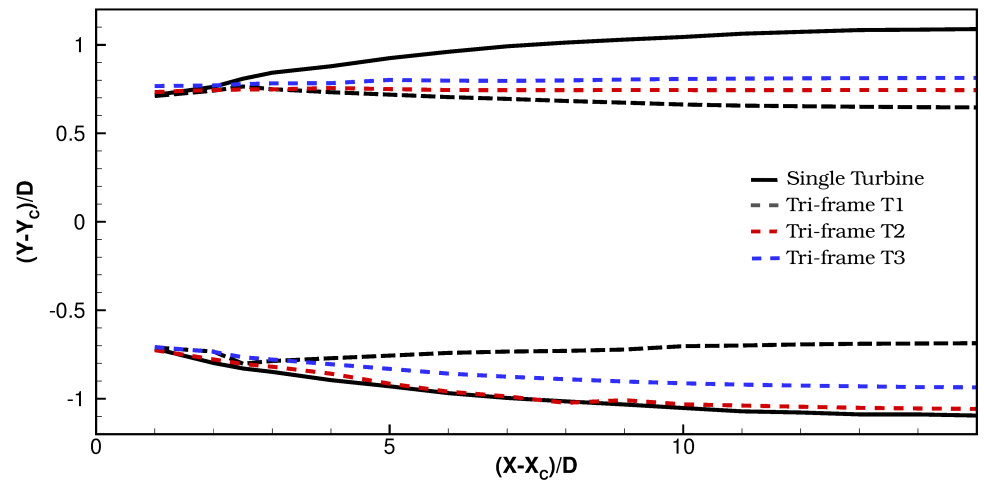

(c)

Figure 16: Special wake function plots for (a) Isolated turbine; (b) T1 turbine in TriFrame; (c) Boundaries of the turbine wakes computed using the locus of maxima of special wake function for all turbines. For turbines T2 and T3, negative ordinate values represent locations close to the wall and positive values are locations close to channel center. 

but T1 turbine wake has dampened TKE levels in the near wake. However, in the far wake (beyond 10D downstream), the TKE levels for all turbines are similar (within 3\%). 


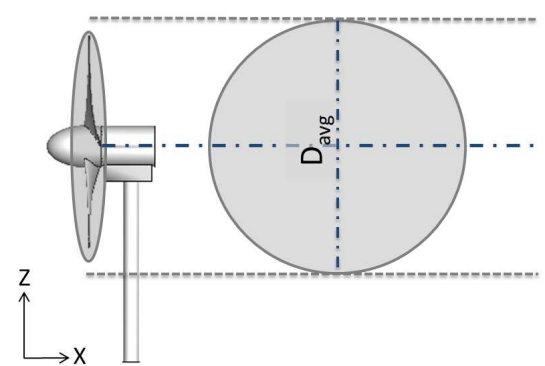

(a)

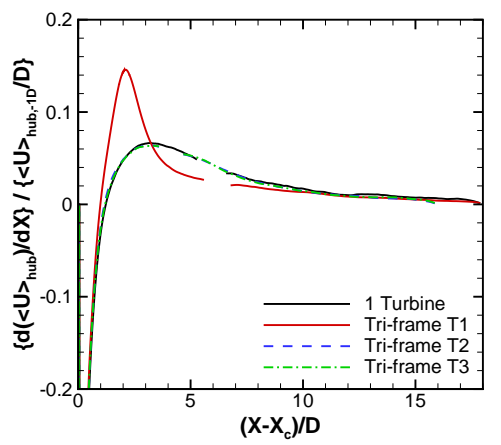

$(\mathrm{c})$

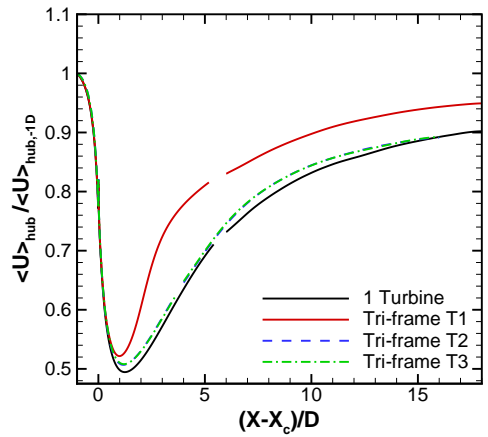

(b)

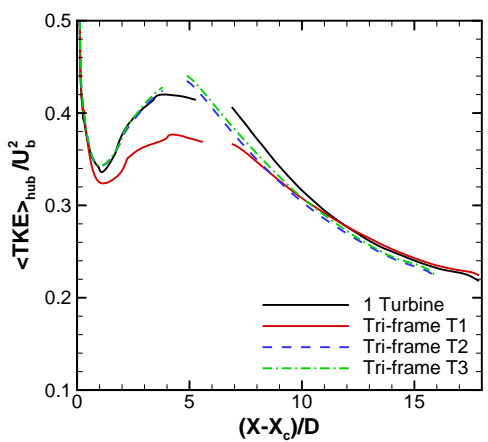

$(\mathrm{d})$

Figure 17: Characteristics of the TriFrame turbine wakes in comparison with the single turbine wake using the disc-averaged quantities. (a) A schematic showing computation of the discaveraged quantities at spanwise-vertical discs with diameter $D_{a v g}>D$ along the rotor axial direction at different streamwise locations, and (b) disc-averaged streamwise velocity $\left(D_{\text {avg }}=\right.$ $1.1 D),(\mathrm{c})$ wake recovery rate computed using the disc-averaged streamwise velocity, and (d) disc-averaged TKE $\left(D_{\text {avg }}=1.4 D\right)$ at different streamwise locations. 


\begin{tabular}{cccccc}
\hline$x$ & $\begin{array}{c}\text { Single } \\
\text { Turbine }\end{array}$ & $\begin{array}{c}\text { TriFrame } \\
\text { T1 }\end{array}$ & $\begin{array}{c}\text { TriFrame } \\
\text { T2,T3 }\end{array}$ & $\frac{P_{T 4}-P_{S T 2}}{P_{S T 2}}$ & $\frac{P_{\Delta 2}-3 \times P_{S T 2}}{P_{\Delta 2}}$ \\
\hline 5D & $69.1 \%$ & $81.0 \%$ & $69.7 \%$ & $37.9 \%$ & $17.0 \%$ \\
8D & $79.1 \%$ & $86.8 \%$ & $80.7 \%$ & $24.7 \%$ & $11.2 \%$ \\
10D & $83.1 \%$ & $89.7 \%$ & $84.4 \%$ & $20.5 \%$ & $8.7 \%$ \\
$15 \mathrm{D}$ & $85.5 \%$ & $93.8 \%$ & $88.8 \%$ & $16.0 \%$ & $4.7 \%$ \\
\hline
\end{tabular}

Table 2: Recovery of velocity in the wakes of different turbines at downstream distances 5D, $8 \mathrm{D}, 10 \mathrm{D}$ and $15 \mathrm{D}$ from the turbine. $P_{T 4}$ and $P_{\Delta 2}$ denote power produced by $\mathrm{T} 4$ turbine and total TriFrame power of $\Delta 2$, respectively, for a second downstream TriFrame in the wake. $P_{\#}$ denotes the power produced by turbine/TriFrame \# as in Fig. 18. (See Appendix A for evaluation of $P_{T 4} / P_{S T 2}$ and $\left.P_{S T 2} / P_{\Delta 2}\right)$

\subsection{TriFrame deployment in an array}

A large scale power producing array of turbines can be constructed by deploying multiple TriFrames of turbines at a site. This means successive TriFrames are in the wake of preceding ones. Since the power production by axial hydrokinetic turbines is proportional to the cube of incoming velocity, a

440 speedier recovery of the wake means better performance for the downstream TriFrames. From the above results of the simulations, it is clear that each of the turbines in the TriFrame has different wake characteristics. Fig. 16 describes the averaged spatial evolution of their wake and differences with single turbine. The flow accelerates between the two downstream turbines resulting in an early recovery of the upstream $\mathrm{T} 1$ turbine compared to the single turbine wake. Table 2 compares the percentage of upstream velocity, averaged over a disc (see Fig. 17(a)), recovered for each of the turbines at distances 5D, 8D, 10D and 15D downstream of turbine. At 5D downstream of the turbine, T1 turbine has recovered $81 \%$ of the incoming flow whereas the isolated turbine wake has recovered only $70 \%$ by this distance. Due to the slowing recovery rate (Fig. $17(\mathrm{c})$ ) it takes another 5D (total of 10D downstream) for the single turbine to recover up to $81 \%$, at which point T1 turbine has recovered approximately $90 \%$ of the upstream value.

Consider two TriFrames placed in an array in an aligned manner as shown in Fig. 18. For the second TriFrame $\Delta 2$, the incoming velocities for T4 is lower than T1 (of first TriFrame $\Delta 1$ ) by fractions listed (as percent) in column 3 of Table 2. Similar fraction for T5 over T2 and T6 over T3 is in the next column. The fifth column in the table $\left(P_{T 4}-P_{S T 2}\right) / P_{S T 2}$ represents the amount of additional power that T4 turbine of TriFrame $\Delta 2$ generates as compared to ST2 turbine a of single turbine array instead of TriFrames $\left(P_{\#}\right.$ denotes the power produced by turbine/TriFrame index \# as shown in Fig. 18). If the distance between the successive units $(x)$ is $5 \mathrm{D}$, the upstream turbine (T4) of second TriFrame $(\Delta 2)$ can generate up to $37.9 \%$ more power. Owing to different velocity recovery, the power production of a single turbine in the wake ( ST2 in 465 Fig. 18(b)) will be different from that of the turbines in array of TriFrames ( T4 - T6 in Fig. 18(a)). The last column in Table 2 gives the error in TriFrame power 

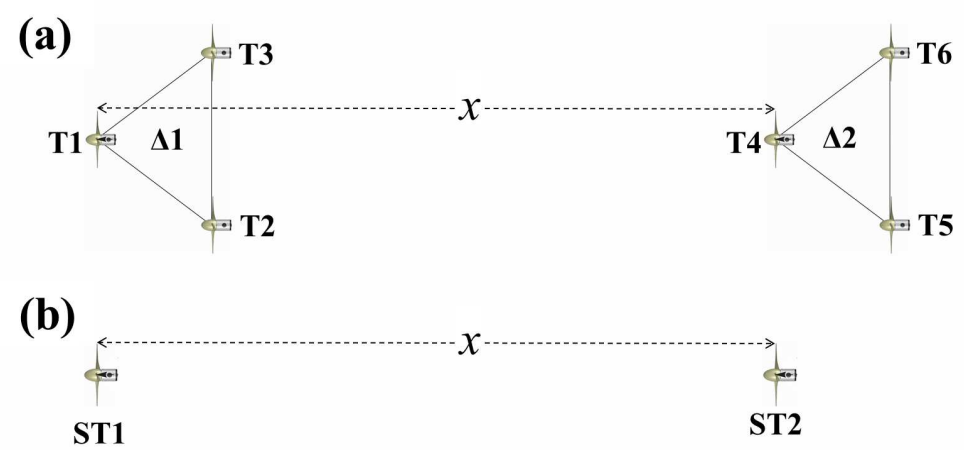

Figure 18: Array of MHK turbines consising of (a) TriFrame configuration with TriFrames $\Delta 1$ and $\Delta 2$ and (b) single turbines ST1 and ST2 separated by $x$ distance.

production estimate (of $\Delta 2$ ) if the individual turbine wakes of the TriFrame $\Delta 1$ were simply modeled as single turbine wakes. If the spacing between successive TriFrames is $5 \mathrm{D}$, this error could be up to $17 \%$. However, if the spacing is large (15D), less than $5 \%$ error in power production results.

The fluctuating components of velocities in the incoming flow are responsible for fatigue loading on the turbine blades. Long term exposure to fatigue loading compromises the structural integrity of the turbine with severely damaging effect on its performance and safety. Therefore, it is important to analyze the 475 turbulence in the incoming flow for the turbines in an array. Even if the incident flow on first turbine (or first TriFrame) of the array has little to no incoming turbulence, the rotating turbine produces significant levels of turbulence for the downstream turbines of the array. In case of a TriFrame of turbines, lower levels of TKE in the wake of T1 turbine were observed as compared to the single

${ }_{480}$ turbine (ST1) wake (see Fig.17(d)). The disc averaged TKE at 5D for T1 wake is $10 \%$ lower than that of the isolated turbine. At $10 \mathrm{D}$, the averaged TKE for all turbines (T1, T2 and T3) are 4\% lower than the ST1 wake whereas at 15D they are within $2.5 \%$ of the corresponding ST1 value. We therefore demonstrate that for an array deployment of MHK turbines, the interaction between wakes has to be properly resolved in a TriFrame simulation to provide a better prediction of generated power. As seen in aforementioned calculations of the power generation estimates (values in Table 2), the power produced by TriFrame of turbines could be underestimated if wake-interaction effects are unaccounted.

\section{Conclusion}

In this paper, the flow past a TriFrame of hydrokinetic turbines in an openchannel was studied using both experiments and numerical simulations. Geometry resolving LES is of special relevance to hydrokinetic turbines because reduced order modeling techniques, such as actuator line/disc methods, cannot predict the wake accurately [8] since they do not model the nacelle and cannot 
495

capture the rich dynamics of the hub vortex. To the best of our knowledge, our work is the first time a geometry resolving simulation was performed for a turbulent flow past multiple hydrokinetic turbines. The computed results were compared with the measurements from the laboratory experiments. The mean velocity and the turbulent statistics were accurately predicted in the wake of the TriFrame. Further analysis of the computed results revealed characteristic features of the TriFrame wake that could not be identified in a single turbine wake. In particular, the two rows of turbines in the TriFrame give rise to different wakes. For the upstream turbine in the TriFrame, the shear layer gets constricted after reaching the second row at 2D downstream distance. This is attributed to the Venturi effect which has been observed earlier in the experiments with wind turbines [13]. Consequently, flow acceleration is obtained in the region between the outer shear layers of the adjacent turbine wakes. The TKE levels are also lower in this region and the momentum deficit recovers faster for the upstream turbine. On comparison with a separate single turbine simulation, it was observed that the wake of the upstream turbine has higher velocity and lower TKE than the single turbine. The two second row turbines, however, produced higher TKE levels in the wake around the tip region in the near wake. In the farther wake after $5 \mathrm{D}$, the TKE levels were similar. The general shape of the three wakes of turbines compared in Fig. 16 showed different ${ }_{15}$ characteristics. Since all three turbines rotate in same direction, the two downstream turbines (T2 and T3) are effected differently, resulting in the spanwise asymmetry of the superwake of the TriFrame (see Fig. 16).

The faster momentum deficit recovery and lower TKE in the wake of the upstream turbine of the TriFrame are advantageous when using the TriFrame assembly to build a large turbine array. Higher TKE levels in some near-wake regions in the wake of the second row turbines will produce undesirable effect of higher fatigue loads on the downstream turbines in the array. If the TriFrames are used to build the array, the power produced by TriFrame is higher than three single turbines operating independently. The amount of excess power generated depends on the inter-TriFrame spacing in the array and increases with decreasing spacing. Therefore, estimating the power production of downstream TriFrame with three single turbines will underestimate the power by amount listed in last column of Table 2. In future work, the 3D resolved flow computations obtained here will be used to construct reduced-order models helpful in finding the optimal Triframe layouts using such techniques as used earlier [27]. Our future work will also focus on performing array level computations with TriFrame configurations in a real-life marine environment.

\section{Appendix A. Calculation of $P_{T 4} / P_{S T 2}$ and $P_{S T 2} / P_{\Delta 2}$ in Table 2}

Power produced by a turbine $P$ is given as:

$$
P=\frac{1}{2} \rho A C_{p} U^{3}
$$


where $C_{p}$ is the power coefficient of the turbine, $\rho$ is density of fluid, $A=$ $0.25 \pi D^{2}$ is area intercepted by turbine and $U$ is the incoming fluid velocity. Assuming all turbines have the same diameter, constant fluid density and are operating at the same power coefficient, for two different incoming velocities $U_{1}$ and $U_{2}$ the power production ratio is given as

$$
\frac{P_{1}}{P_{2}}=\left(\frac{U_{1}}{U_{2}}\right)^{3}
$$

Consider two units in an array of single turbines such that one turbine is placed in wake of another. The incoming velocity $U_{h u b,-1 D}$ for preceding turbine and $U_{h u b,-1 D}^{(2)}$ for the next downstream turbine in array are related by $U_{h u b,-1 D}^{(2)}=R * U_{h u b,-1 D}$ where $R$ and $U_{h u b,-1 D}$ are recovery fraction (shown as $\%$ in Table 2 or in Fig. 17(b)) and incoming velocity for the preceding turbine (ST1) in the upstream unit. Same relationship holds true for similarly positioned turbines in two TriFrame units for an array of TriFrames. $U_{h u b,-1 D}$ for different upstream turbines are listed in Table A.3. Using the above relation (in

\begin{tabular}{ccccc}
\hline & Single Turine $(\mathrm{ST} 1)$ & TriFrame T1 & TriFrame T2 & TriFrame T3 \\
\hline$U_{h u b,-1 D} / U_{b}$ & 1.135 & 1.135 & 1.123 & 1.126 \\
\hline
\end{tabular}

Table A.3: Incoming velocity for upstream TriFrame turbines or single turbine.

Eq. A.2) for T4 as 1 and ST2 as 2 we can write,

$$
\begin{aligned}
\frac{P_{T 4}}{P_{S T 2}} & =\left(\frac{R_{T 1} U_{h u b,-1 D, T 1}}{R_{S T 1} U_{h u b,-1 D, S T 1}}\right)^{3} \\
& =\left(\frac{R_{T 1} \times 1.135 U_{b}}{R_{S T 1} \times 1.135 U_{b}}\right)^{3} \\
& =\left(\frac{R_{T 1}}{R_{S T 1}}\right)^{3}
\end{aligned}
$$

and

$$
\begin{aligned}
\frac{P_{S T 2}}{P_{\Delta 2}} & =\frac{P_{S T 2}}{P_{T 4}+P_{T 5}+P_{T 6}} \\
& =\frac{\left(R_{S T 1} U_{h u b,-1 D, S T 1}\right)^{3}}{\left(R_{T 1} U_{h u b,-1 D, T 1}\right)^{3}+\left(R_{T 2} U_{h u b,-1 D, T 2}\right)^{3}+\left(R_{T 3} U_{h u b,-1 D, T 3}\right)^{3}} \\
& =\frac{\left(R_{S T 1} \times 1.135 U_{b}\right)^{3}}{\left(R_{T 1} \times 1.135 U_{b}\right)^{3}+\left(R_{T 2} \times 1.123 U_{b}\right)^{3}+\left(R_{T 3} \times 1.126 U_{b}\right)^{3}} \\
& =\frac{\left(1.135 R_{S T 1}\right)^{3}}{\left(1.135 R_{T 1}\right)^{3}+\left(1.123 R_{T 2}\right)^{3}+\left(1.126 R_{T 3}\right)^{3}}
\end{aligned}
$$

Columns four and five in Table 2 are populated using the above relations A.3 and A.4, respectively, and the values of recovery $(\mathrm{R})$ at respective downstream distances in the same table. 


\section{Acknowledgments}

This work was supported by NSF grant IIP-1318201. Computational re${ }_{540}$ sources were provided by the University of Minnesota Supercomputing Institute.

\section{References}

[1] W. Batten, A. Bahaj, A. Molland, J. Chaplin, The prediction of the hydrodynamic performance of marine current turbines, Renewable energy 33 (5) (2008) 1085-1096.

[2] M. J. Lawson, Y. Li, D. C. Sale, Development and verification of a computational fluid dynamics model of a horizontal-axis tidal current turbine, in: ASME 2011 30th International Conference on Ocean, Offshore and Arctic Engineering, American Society of Mechanical Engineers, 2011, pp. 711-720.

[3] A. Bahaj, L. Myers, R. Rawlinson-Smith, M. Thomson, The effect of boundary proximity upon the wake structure of horizontal axis marine current turbines, Journal of Offshore Mechanics and Arctic Engineering 134 (2) (2012) 021104.

[4] S. Kang, F. Sotiropoulos, Numerical modeling of 3D turbulent free surface flow in natural waterways, Advances in Water Resources 40 (2012) 23-36.

[5] G. Pinon, P. Mycek, G. Germain, E. Rivoalen, Numerical simulation of the wake of marine current turbines with a particle method, Renewable Energy 46 (2012) 111-126.

[6] L. Chamorro, C. Hill, S. Morton, C. Ellis, R. Arndt, F. Sotiropoulos, On the interaction between a turbulent open channel flow and an axial-flow turbine, Journal of Fluid Mechanics 716 (2013) 658-670.

[7] I. Afgan, J. McNaughton, S. Rolfo, D. Apsley, T. Stallard, P. Stansby, Turbulent flow and loading on a tidal stream turbine by LES and RANS, International Journal of Heat and Fluid Flow 43 (2013) 96-108.

[8] S. Kang, X. Yang, F. Sotiropoulos, On the onset of wake meandering for an axial flow turbine in a turbulent open channel flow, Journal of Fluid Mechanics 744 (2014) 376-403.

[9] L. Chamorro, C. Hill, V. Neary, B. Gunawan, R. Arndt, F. Sotiropoulos, Effects of energetic coherent motions on the power and wake of an axial-flow turbine, Physics of Fluids (1994-present) 27 (5) (2015) 055104.

[10] T. Stallard, T. Feng, P. Stansby, Experimental study of the mean wake of a tidal stream rotor in a shallow turbulent flow, Journal of Fluids and Structures 54 (2015) 235-246. 
[11] I. Ammara, C. Leclerc, C. Masson, A viscous three-dimensional differential/actuator-disk method for the aerodynamic analysis of wind farms, Journal of Solar Energy Engineering 124 (4) (2002) 345-356.

[12] M. Calaf, C. Meneveau, J. Meyers, Large eddy simulation study of fully developed wind-turbine array boundary layers, Physics of Fluids (1994present) 22 (1) (2010) 015110.

[13] L. P. Chamorro, R. Arndt, F. Sotiropoulos, Turbulent flow properties around a staggered wind farm, Boundary-layer meteorology 141 (3) (2011) 349-367.

[14] X. Yang, S. Kang, F. Sotiropoulos, Computational study and modeling of turbine spacing effects in infinite aligned wind farms, Physics of Fluids (1994-present) 24 (11) (2012) 115107.

[15] X. Yang, F. Sotiropoulos, R. J. Conzemius, J. N. Wachtler, M. B. Strong, Large-eddy simulation of turbulent flow past wind farms in complex terrains: The virtual wind simulator (VWiS), Wind Energy 16 (1) (2013) $1-20$.

[16] L. Myers, A. Bahaj, An experimental investigation simulating flow effects in first generation marine current energy converter arrays, Renewable Energy 37 (1) (2012) 28-36.

[17] T. Daly, L. E. Myers, A. S. Bahaj, Experimental investigation of the effects of the presence and operation of tidal turbine arrays in a split tidal channel, Volume 9 Marine and Ocean Technology (2011) 2262.

[18] T. Stallard, R. Collings, T. Feng, J. Whelan, Interactions between tidal turbine wakes: experimental study of a group of three-bladed rotors, Philosophical Transactions of the Royal Society of London A: Mathematical, Physical and Engineering Sciences 371 (1985) (2013) 20120159.

[19] S. C. James, E. Seetho, C. Jones, J. Roberts, Simulating environmental changes due to marine hydrokinetic energy installations, in: OCEANS 2010, IEEE, 2010, pp. 1-10.

[20] M. Harrison, W. Batten, A. Bahaj, A blade element actuator disc approach applied to tidal stream turbines, OCEANS 2010 (2010) 1-8.

[21] R. Malki, I. Masters, A. J. Williams, T. N. Croft, Planning tidal stream turbine array layouts using a coupled blade element momentumcomputational fluid dynamics model, Renewable Energy 63 (2014) 46-54.

[22] J. A. Colby, M. A. Adonizio, Hydrodynamic analysis of kinetic hydropower arrays, Waterpower XVI 204. 
[23] L. Bai, R. R. Spence, G. Dudziak, Investigation of the influence of array arrangement and spacing on tidal energy converter (TEC) performance using a 3-dimensional CFD model, in: Proceedings of the 8th European Wave and Tidal Energy Conference, Uppsala, Sweden, 2009, pp. 654-660.

[24] A. Olczak, T. Stallard, T. Feng, P. Stansby, Comparison of a rans blade element model for tidal turbine arrays with laboratory scale measurements of wake velocity and rotor thrust, Journal of Fluids and Structures 64 (2016) 87-106.

[25] M. J. Churchfield, Y. Li, P. J. Moriarty, A large-eddy simulation study of wake propagation and power production in an array of tidal-current turbines, Philosophical Transactions of the Royal Society A: Mathematical, Physical and Engineering Sciences 371 (1985) (2013) 20120421.

[26] X. Yang, S. Kang, F. Sotiropoulos, Toward a simulation-based approach for optimizing MHK turbine arrays in natural waterways, in: Proceedings of the 1st Marine Energy Technology Symposium, 2013.

[27] P. Stansby, T. Stallard, Fast optimisation of tidal stream turbine positions for power generation in small arrays with low blockage based on superposition of self-similar far-wake velocity deficit profiles, Renewable Energy 92 (2016) 366-375.

[28] L. Ge, F. Sotiropoulos, A numerical method for solving the 3D unsteady incompressible navier-stokes equations in curvilinear domains with complex immersed boundaries, Journal of Computational Physics 225 (2) (2007) 1782-1809.

[29] S. Kang, A. Lightbody, C. Hill, F. Sotiropoulos, High-resolution numerical simulation of turbulence in natural waterways, Advances in Water Resources 34 (1) (2011) 98-113.

[30] J. Smagorinsky, General circulation experiments with the primitive equations: I. the basic experiment*, Monthly weather review 91 (3) (1963) 99-164.

[31] M. Germano, U. Piomelli, P. Moin, W. H. Cabot, A dynamic subgrid-scale eddy viscosity model, Physics of Fluids A: Fluid Dynamics (1989-1993) 3 (7) (1991) 1760-1765.

[32] L. Ge, F. Sotiropoulos, 3D unsteady RANS modeling of complex hydraulic engineering flows. I: Numerical model, Journal of Hydraulic Engineering 131 (9) (2005) 800-808.

[33] H. Werner, H. Wengle, Large-eddy simulation of turbulent flow over and around a cube in a plate channel, in: Turbulent Shear Flows 8, Springer, 1993, pp. 155-168. 
[34] J.-I. Choi, R. C. Oberoi, J. R. Edwards, J. A. Rosati, An immersed boundary method for complex incompressible flows, Journal of Computational Physics 224 (2) (2007) 757-784.

[35] Y. Saad, M. H. Schultz, Gmres: A generalized minimal residual algorithm for solving nonsymmetric linear systems, SIAM Journal on scientific and statistical computing 7 (3) (1986) 856-869.

[36] C. Hill, M. Musa, L. P. Chamorro, C. Ellis, M. Guala, Local scour around a model hydrokinetic turbine in an erodible channel, Journal Hydraulic Engineering 140 (8) (2014) 04014037.

[37] C. Hill, M. Musa, M. Guala, Interaction between instream axial flow hydrokinetic turbines and uni-directional flow bedforms, Renewable Energy 86 (2016) 409-421.

660

[38] C. Hill, J. Kozarek, F. Sotiropoulos, M. Guala, Hydrodynamics and sediment transport in a meandering channel with a model axial-flow hydrokinetic turbine, Water Resources Research 52 (2) (2016) 860-879. 